\title{
Differential feeding as a concept for the realization of broadband dual- polarized antennas with very high polarization purity
}

\author{
Grzegorz Adamiuk*, Werner Wiesbeck and Thomas Zwick \\ (1) Institut für Hochfrequenztechnik und Elektronik, University of Karlsruhe, \\ Germany, Kaiserstr. 12, 76131, Karlsruhe, Germany \\ E-mail: grzegorz.adamiuk@ihe.uka.de
}

\section{Introduction}

The latest research on Ultra Wideband (UWB) Technology showed an increasing demand on the ultra wideband dual-polarized antenna elements [1]. In the literature only few antennas are available that allow for polarization diversity in the impulse-based UWB systems. Most of them radiate the wave over very wide bandwidth using the traveling wave principle like the well known quad ridged horn antenna, the dielectric rod antenna [2] or the vivaldi antenna [3]. However such antennas are generally bulky and are difficult to integrate into any device. For this reason a compact antenna in planar technology radiating perpendicularly to the antenna surface is needed. A very important demand on such antenna is a common phase center of radiation for both polarizations, which is stable over the very wide bandwidth. This paper introduces a novel concept for the realization of the antenna meeting the mentioned requirements. The antenna principle and feeding scheme is described. Subsequently the prototype and measurement results are presented.

\section{Antenna principle}

The layout of the antenna is shown in Fig.1. It is fed by the four microstrip lines, which feed the four monopole radiators. On the bottom side of the antenna a circle is cut out from the ground plane. The antenna is fully symmetrical considering the center point of the antenna as the point of symmetry. Such an arrangement assures the same radiation properties for both polarizations in the equivalent planes.

In order to radiate the single polarization the antenna has to be fed at two ports. Accordingly to Fig.1 the pairs of ports 1.1-1.2 and 2.1-2.2 have to be fed for horizontal and vertical polarization, respectively. In the case, where the ports are fed by the same signals considering amplitude and phase, the antenna behaves as two monopole radiators placed in the close proximity from each other. Such an excitation results in two main beams in one half-space of the antenna and poor polarization purity. The antenna reveals its advantages when the signals fed to the appropriate ports are out-of-phase. In such a case the signals fed to the oppositely lying ports have to be equal in the amplitude and the phase difference must be 

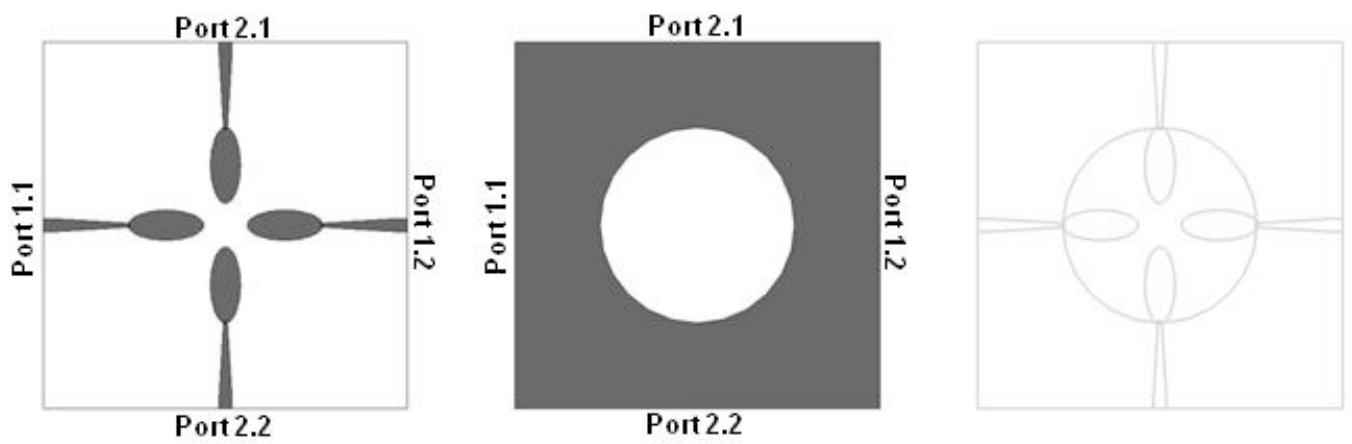

Fig.1 Layout of the antenna (left: top view, center: bottom view, right: transparent)

exactly 180 degree for each frequency over the desired bandwidth. Such an excitation is called differential feeding of the antenna.

The schematical electric field distribution in the antenna fed at the ports 1.1 and 1.2 with the differential signals is shown in Fig.2. On the left side the cross section through the antenna is introduced. As can be noticed the orientations of the electric field vectors at the feeding points are opposite, which yields the phase shift between signals of 180 degree. The upper side introduces the plane with the monopole radiators and the bottom side the ground plane with the circle. As can be concluded from the scheme the waves coming from the ports interfere in the middle of the structure constrictively. The signals are merged with each other and since the radiation conditions are fulfilled, the wave is emitted into the top and bottom of the antenna surface.

The two differentially fed monopoles excite a certain mode in the circle, of which order depends on the frequency and geometrics of the antenna. The schematical electric field distribution of the base mode is introduced in Fig. 2 on the right side. The resulting radiation pattern is adequate to the field distribution in the circle. The mode introduced in Fig. 2 creates one main beam in one half-space of the antenna. The excitation of the higher modes results in the split of the main beam. The type of the mode excited in the antenna is frequency dependent and for this reason the dimensions of the antenna are closely linked with the shape of the radiation pattern. Firstly the dimensions must be large enough in order to radiate the lowest desired frequency. On the other hand they should be small enough in order not to excite the higher modes, which deform the radiation pattern. The excitation of the higher modes introduces a limitation for the antenna considering the usability of the radiation pattern. However the differential concept of the excitation allows for the applicability of the antenna in the relative bandwidth of approx. $100 \%$, which is enough for the UWB technology. 

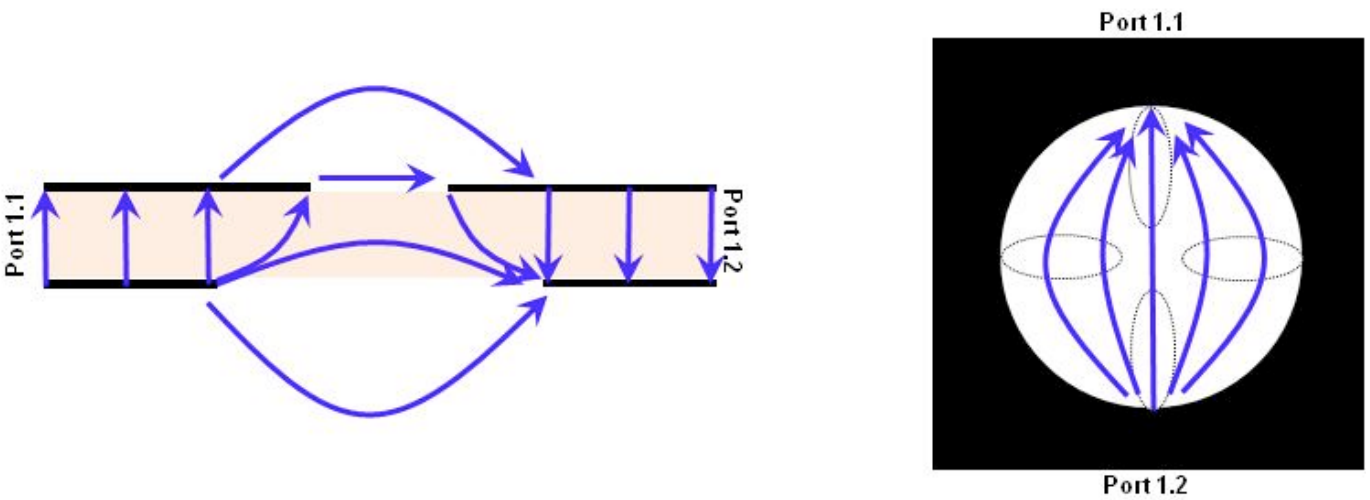

Fig.2 Schematical electric field distribution in the antenna (left: cross section, right: bottom view)

Another advantage of the simultaneous excitation at both ports is the fact, that the phase center of radiation does not change its position. Regardless of the excited mode or the frequency, the phase center is always in the middle of the structure. The point, as can be concluded from the drawings, is the same for both polarizations. The alignment of the phase centers for both polarizations is an important issue. The offset between that points leads to the errors in the imaging, radar and localization systems, which cannot be always compensated by the algorithms.

It can be noticed, that the electric field vectors at the microstrip lines for the orthogonal polarization are oriented transversally to the line. Such a field distribution is not able to excite a mode that is able to propagate in the microstrip line. This results in an outstanding decoupling of the ports of the antenna.

\section{Antenna Prototype and Results}

The antenna was designed for the FCC UWB frequency range from $3.1 \mathrm{GHz}$ to 10.6 GHz. The photo of the fabricated device can be seen in Fig.3a). It was etched on the substrate Duroid 5880 with the thickness $0.79 \mathrm{~mm}$. The radiation characteristics of the antenna were measured in the anechoic chamber with the VNA. The appropriate ports of the antenna were connected with the outputs of the differential $3 \mathrm{~dB}$ power divider by the $50 \mathrm{~cm}$ long microwave cables. The differential $3 \mathrm{~dB}$ power divider used in the measurement bases on the same principle as introduced in [4]. The input impedance matching of such arranged structure was lower than $-10 \mathrm{~dB}$ and the coupling between ports lower than $-30 \mathrm{~dB}$ in the designated frequency range.

The measured gain for single polarization in the E-plane for Co- and X-polarization is introduced in Fig.3b) and Fig.3c), respectively. It can be observed that for the copolarization the antenna radiates with two beams oriented oppositely to each other, as explained before theoretically. The radiation pattern remains relatively constant in the useful frequency range from $3 \mathrm{GHz}$ to $10 \mathrm{GHz}$. The cross polarized components are very weak in comparison to the co-polarized (please note the scale). In the rear half-space of the antenna (absolute angles higher than 90 degree) the stronger radiation in cross- 


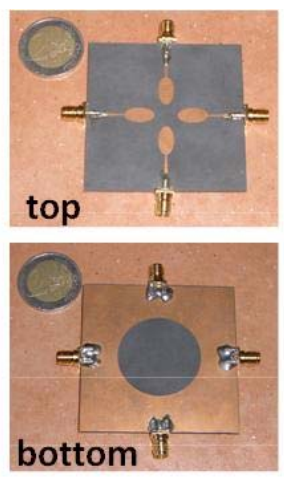

a)

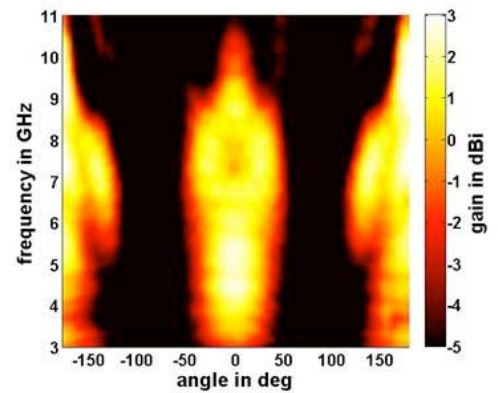

b)

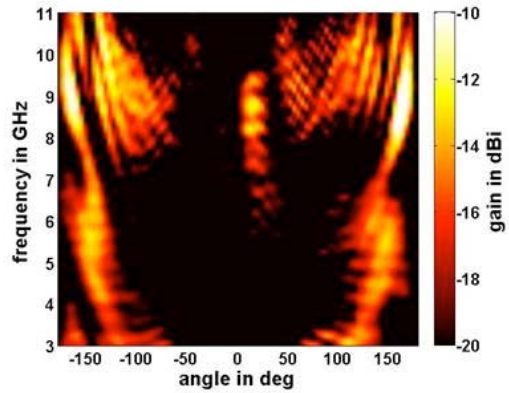

c)

Fig. 3 a) Photo of the prototype antenna

b) Measured gain for one set of ports (E-plane, $\mathrm{Co}-\mathrm{Pol})$

c) Measured gain for one set of ports (E-Plane, X-Pol)

polarization can be observed. The investigations have shown, that the radiation comes from the feeding microstrip lines, which are tapered. However the polarization decoupling in the direction 0 degree is approx. $25 \mathrm{~dB}$ for the whole frequency range.

\section{Summary}

In this paper a new concept for the design of the ultra broadband antennas with dualorthogonal, linear polarization is introduced. It enables an outstanding polarization decoupling over the very wide frequency range. The antenna built accordingly to this concept can be realized in the planar technology, where the radiation occurs perpendicularly to the antenna surface. This feature is not common in the few dualpolarized ultra wideband antennas introduced in the literature. The concept assures that the phase center of radiation is the same for both polarizations and does not move at all in the whole frequency range. This is very advantageous especially for the impulse-based UWB systems. The coupling between ports is very low. The measurements with the antenna basing on this concept confirm the theoretical assumptions and show very good performance of the device.

\section{References}

[1] R.Zetik, J.Sachs, R.Thomä, "UWB Short Range Radar Sensing” IEEE Instrumentation and Measurement Magazine, Vol. 10, pp. 39-45, April 2007.

[2] J.Chung, C.Chen, " Two-Layer Dielectric Rod Antenna ", IEEE Transactions on Antennas and Propagation, vol. 56, issue 6, pp. 1541-1547, June 2008

[3] G.Adamiuk, T.Zwick, W.Wiesbeck, "Dual-orthogonal polarized vivaldi antenna for ultra wideband applications", Proceedings of the XVII International Conference on Microwaves, Radar and Wireless Communications, vol. 2, pp. 282-285, Wroclaw, Poland, May

[4] M.E.Bialkowski, A.M.Abbosh, " Design of a Compact UWB Out-of-Phase Power Divider", IEEE Microwave and Wireless Components Letters, vol. 17, issue 4, pp.289-291, April 2007. 